Gene Regulation:

A Tandem SH2 Domain in Transcription

Elongation Factor Spt6 Binds the

Phosphorylated RNA Polymerase II

C-terminal Repeat Domain (CTD)

Mai Sun, Laurent Larivière, Stefan Dengl,

Andreas Mayer and Patrick Cramer

J. Biol. Chem. 2010, 285:41597-41603.

doi: 10.1074/jbc.M110.144568 originally published online October 6, 2010

Access the most updated version of this article at doi: 10.1074/jbc.M110.144568

Find articles, minireviews, Reflections and Classics on similar topics on the JBC Affinity Sites.

Alerts:

- When this article is cited

- When a correction for this article is posted

Click here to choose from all of JBC's e-mail alerts

This article cites 43 references, 20 of which can be accessed free at http://www.jbc.org/content/285/53/41597.full.html\#ref-list-1 


\title{
A Tandem SH2 Domain in Transcription Elongation Factor Spt6 Binds the Phosphorylated RNA Polymerase II C-terminal Repeat Domain (CTD)*
}

Received for publication, May 13, 2010, and in revised form, September 6, 2010 Published, JBC Papers in Press, October 6, 2010, DOI 10.1074/jbc.M110.144568

\author{
Mai Sun, Laurent Larivière, Stefan Dengl, Andreas Mayer, and Patrick Cramer
}

From the Gene Center Munich and Department of Biochemistry, Center for Integrated Protein Science Munich (CIPSM), Ludwig-Maximilians-Universität München, Feodor-Lynen-Strasse 25, 81377 Munich, Germany

Spt6 is an essential transcription elongation factor and histone chaperone that binds the C-terminal repeat domain (CTD) of RNA polymerase II. We show here that Spt6 contains a tandem SH2 domain with a novel structure and CTD-binding mode. The tandem $\mathrm{SH} 2$ domain binds to a serine 2-phosphorylated CTD peptide in vitro, whereas its $\mathrm{N}$-terminal SH2 subdomain, which we previously characterized, does not. CTD binding requires a positively charged crevice in the $\mathrm{C}$-terminal SH2 subdomain, which lacks the canonical phospho-binding pocket of $\mathrm{SH} 2$ domains and had previously escaped detection. The tandem SH2 domain is apparently required for transcription elongation in vivo as its deletion in cells is lethal in the presence of 6-azauracil.

Spt6 is an essential nuclear protein $(1,2)$ that is required for transcription elongation together with $\operatorname{Spt} 4$ and $\operatorname{Spt} 5(3,4)$. Spt6 co-localizes with Spt5 on transcribed genes in Drosophila polytene chromosomes $(5,6)$. Spt6 stimulates the elongation rate of RNA polymerase II in vivo (7). There is strong evidence that Spt6 is a histone chaperone. In cells lacking functional Spt6, transcription can start from cryptic promoters within the coding regions of genes (8), and transcription reinitiation from regular promoters is facilitated (9). This suggests that Spt6 is required for the correct positioning of nucleosomes in transcribed regions. Consistently, Spt6 interacts with histone $\mathrm{H} 3$ and can deposit nucleosomes on DNA (10). Spt6 is further involved in mRNA 3'-end formation (11) and export (12).

The C-terminal region of mouse Spt6 binds the Ser-2phosphorylated CTD, ${ }^{2}$ suggesting a mechanism for how Spt6 is recruited to elongating RNA polymerase II (12). The Spt6 C-terminal region was predicted to contain an $\mathrm{SH} 2$ domain. We recently reported the crystal structure of this $\mathrm{SH} 2$ domain (13), which is the only SH2 domain in the

\footnotetext{
* This work was supported by grants from the Deutsche Forschungsgemeinschaft, the Sonderforschungsbereich SFB646, the Transregio 5, the Nanosystems Initiative Munich (NIM) the Elitenetzwerk Bayern, the Fonds der Chemischen Industrie, and the Jung Stiftung.

The atomic coordinates and structure factors (code 3PJP) have been deposited in the Protein Data Bank, Research Collaboratory for Structural Bioinformatics, Rutgers University, New Brunswick, NJ (http://www.rcsb.org/).

${ }^{1}$ To whom correspondence should be addressed. Tel.: 49-89-2180-76951; Fax: 49-89-2180-76999; E-mail: cramer@LMB.uni-muenchen.de.

${ }^{2}$ The abbreviations used are: CTD, C-terminal repeat domain; 6-AU, 6-azauracil.
}

yeast genome (14). The structure contained residues 12511349 of Candida glabrata Spt6 and showed a fold that combined features of both $\mathrm{SH} 2$ domain subfamilies present in higher eukaryotes $(15,16)$, likely resembling a common evolutionary ancestor of all SH2 domains (13). The structure also revealed a shallow phospho-binding pocket that explained the unusual binding specificity for phospho-serine rather than phospho-tyrosine. Our previous work, however, also showed that the SH2 domain alone did not bind CTD phospho-peptides (13), although a more extended C-terminal region does (12).

To resolve this discrepancy, we investigated the structure and CTD binding of C-terminal fragments of Spt6 encompassing the $\mathrm{SH} 2$ domain and additional flanking regions. We found that a second $\mathrm{SH} 2$ domain is present directly $\mathrm{C}$-terminal of the known $\mathrm{SH} 2$ domain. X-ray crystallography revealed that the two SH2 domains form an extended interface and are arranged in a previously unobserved manner into a rigid "tandem SH2 domain." In contrast to the previously studied single $\mathrm{SH} 2$ domain, the tandem $\mathrm{SH} 2$ domain binds Ser-2-phosphorylated CTD peptides in vitro. The tandem SH2 domain is apparently also required for transcription elongation in vivo as shown by 6-azauracil (6-AU) phenotyping. Our results reveal a novel CTD-binding protein module and indicate that the structure and CTD binding function of the Spt6 C-terminal region is conserved in eukaryotes.

\section{EXPERIMENTAL PROCEDURES}

Preparation of the Spt6 Tandem SH2 Domain-DNA containing the tandem SH2 domain of C. glabrata Spt6 (residues 1250-1444) was amplified by polymerase chain reaction from genomic DNA and was cloned into expression vector $\mathrm{pET} 28 \mathrm{~b}(+)$ (Novagen) using restriction sites NdeI and NotI. This results in an open reading frame that encodes the tandem SH2 domain with an $\mathrm{N}$-terminal hexahistidine tag with a thrombin cleavage site. The sequence was modified by the introduction of codons for methionine residues at the positions of residues Leu-1309, Leu-1317, and Leu-1411 by the rapid PCR site-directed mutagenesis (17). The resulting variant was used for selenomethionine labeling as described (18, 19). The tandem SH2 domain was expressed overnight at $18{ }^{\circ} \mathrm{C}$ in Escherichia coli BL21 CodonPlus(DE3)RIL strain cells (Stratagene). For selenomethionine labeling, we used E. coli strain B834(DE3)pLysS (Novagen). Cells were harvested, resuspended in $50 \mathrm{~mm}$ HEPES, pH 7.0, $1 \mathrm{~m} \mathrm{NaCl}, 10 \mathrm{~mm} \beta$-mer- 
captoethanol, $10 \mathrm{~mm}$ imidazole, and lysed by sonication. Cell lysates were subjected to affinity chromatography on a nickelnitrilotriacetic acid column (Qiagen) followed by cleavage of the hexahistidine tag with thrombin and dialysis overnight at $4{ }^{\circ} \mathrm{C}$. The tag and the undigested protein were removed on a second nickel-nitrilotriacetic acid column. DNA was removed by cation exchange chromatography (Mono S 10/100 GL, Amersham Biosciences). After gel filtration (Superose 12 10/ $300 \mathrm{GL}$, Amersham Biosciences), the sample was concentrated to $14.5 \mathrm{mg} / \mathrm{ml}$ and stored at $4{ }^{\circ} \mathrm{C}$ in $50 \mathrm{~mm}$ HEPES, $\mathrm{pH}$ 7.0, $100 \mathrm{~mm} \mathrm{NaCl}, 5 \mathrm{~mm}$ DTT.

Crystal Structure Determination-The purified native and selenomethionine-labeled Spt6 tandem SH2 domain was crystallized by vapor diffusion using 22-24\% PEG 8000, $50 \mathrm{~mm}$ MES, pH 6.5-7, $0.2 \mathrm{M}$ sodium acetate as reservoir solution. Complete diffraction data at 1.7 and $1.6 \AA$ resolution were obtained from crystals of the selenomethionine-labeled protein and unlabeled protein, respectively, and were processed by XDS and scaled by XSCALE (20). The structure was solved by multiwavelength anomalous diffraction using SOLVE, and density modification was performed with RESOLVE (21). The model was automatically built by the program ARP/wARP (22) with some manual rebuilding using Coot (23) and refined with program PHENIX (24). We added water molecules initially with Coot when a peak height greater than $3.0 \sigma$ was obtained in Fourier difference maps. We added more water molecules manually and further refined the model with PHENIX using bulk solvent scaling and individual isotropic $B$-factors. Refinement statistics are shown in Table 1.

Phosphopeptide Interaction Assay-For fluorescence anisotropy measurements, we used a HORIBA Jobin-Yvon Fluoromax 3 spectrofluorometer. The CTD phospho-peptides were synthesized by PANATecs GmbH (Tübingen), had the sequences P1 = SYpSPTSPSYpSPTSPS (pS, phospho-serine), P2 = SYpSPTSPSYSPTSPS, and P3 = SYSPTSPSYPSPTSPS, $\mathrm{P} 4=$ SYSPTSPSYSPTSPS, and were N-terminally labeled with fluorescein aminocaproic acid. The phospho-peptide (500 nM) was dissolved in $20 \mathrm{~mm}$ HEPES, pH 8.0, $10 \mathrm{~mm} \mathrm{NaCl}$, $5 \mathrm{~mm}$ DTT and was titrated at $20^{\circ} \mathrm{C}$ with pure tandem SH2 domain or its mutant variants R1281K, K1434R, or R1281K/ K1434R, and the fluorescence anisotropy was measured after 2 min when equilibrium was reached. Data were analyzed by non-linear least squared regression against $A=A_{0}+\Delta A \times$ $0.5 \times\left(b-\left(b^{2}-\mathrm{P} \times \mathrm{L}\right)^{1 / 2}\right)$ with $b=\mathrm{P}+\mathrm{L}+K_{D}\left(K_{D}\right.$, apparent dissociation constant; P, protein concentration; L, ligand concentration).

Yeast Strains-Saccharomyces cerevisiae strains containing a C-terminal tandem affinity purification-tagged version of Spt6 and the same strain lacking amino acid residues 1250 1451 of Spt6 were as described (13).

\section{RESULTS}

Spt6 Contains a Tandem SH2 Domain-When we examined the Spt6 amino acid sequences flanking the known SH2 domain, we noted additional predicted secondary structure elements C-terminal to the known SH2 domain. In particular, the arrangement of predicted $\alpha$-helices and
TABLE 1

X-ray diffraction data and refinement statistics for the Spt6 tandem SH2 domain

r.m.s., root mean square.

\begin{tabular}{|c|c|c|c|c|}
\hline Crystal & \multicolumn{2}{|c|}{ Se-Met } & & Native \\
\hline \multicolumn{5}{|l|}{ Data collection } \\
\hline Space group & \multirow{2}{*}{\multicolumn{3}{|c|}{$\begin{array}{l}\mathrm{C} 2 \\
109.2,57.5,85.1\end{array}$}} & \multirow{3}{*}{$\begin{array}{l}\mathrm{C} 2 \\
111.2,57.0,88.0\end{array}$} \\
\hline \multirow{2}{*}{ Unit cell dimensions $(\AA)$} & & & & \\
\hline & Peak & Inflection & Remote & \\
\hline Wavelength $(\AA)$ & 0.97958 & 0.97964 & 0.90784 & 0.91881 \\
\hline Resolution $(\AA)$ & $71.1-1.70$ & $54.9-1.72$ & $54.9-1.72$ & $40-1.6$ \\
\hline & $(1.74-1.70)$ & $(1.74-1.72)$ & $(1.76-1.72)$ & $(1.64-1.60)$ \\
\hline$R_{\mathrm{sym}}(\%)$ & $3.4(51.2)$ & $2.9(53.6)$ & $3.1(55.0)$ & $3.2(40.5)$ \\
\hline$I / \sigma I$ & $14.4(2.1)$ & $15.2(2.0)$ & $15.2(2.0)$ & $13.9(2.5)$ \\
\hline Completeness (\%) & 95.7 (97.9) & $95.7(97.6)$ & $95.6(97.2)$ & $95.0(95.6)$ \\
\hline Redundancy & $2.43(2.41)$ & $2.43(2.41)$ & $2.45(2.49)$ & $2.12(2.17)$ \\
\hline \multicolumn{5}{|l|}{ Refinement } \\
\hline Resolution $(\AA)$ & & & & $35-1.6$ \\
\hline Unique reflections & & & & 56,064 \\
\hline$R_{\text {work }} / R_{\text {free }}(\%)$ & & & & $19.8 / 24.0$ \\
\hline \multicolumn{5}{|l|}{ No. of atoms } \\
\hline Protein & & & & 3351 \\
\hline Water & & & & 484 \\
\hline Ligand/Ion & & & & 28 \\
\hline \multicolumn{5}{|l|}{$B$-factors $\left(\AA^{2}\right)$} \\
\hline Protein & & & & 25.8 \\
\hline Water & & & & 37.8 \\
\hline \multicolumn{5}{|l|}{ r.m.s. deviations } \\
\hline Bond lengths $(\AA)$ & & & & 0.006 \\
\hline Bond angles $\left(^{\circ}\right)$ & & & & 0.994 \\
\hline
\end{tabular}

$\beta$-strands suggested that a second $\mathrm{SH} 2$ domain could be present that had escaped detection by standard domain predication procedures. To investigate this, we prepared a recombinant variant of the Spt6 C-terminal region that spanned the known $\mathrm{SH} 2$ domain and the predicted $\mathrm{SH} 2$ domain (see "Experimental Procedures"). Soluble protein was purified and crystallized. The $\mathrm{x}$-ray structure was determined by multiwavelength anomalous diffraction and refined at $1.6 \AA$ resolution (Table 1 ).

The structure revealed the previously reported $\mathrm{SH} 2$ domain (13) and additionally a second SH2 domain (Fig. 1). The two domains, designated hereafter subdomains SH2$\mathrm{N}$ and SH2-C, intimately pack against each other. Therefore, we refer to this structure as tandem SH2 domain. The C-terminal helix of subdomain $\mathrm{SH} 2-\mathrm{N}$ is composed of two parts ( $\alpha \mathrm{B} 1$ and $\alpha \mathrm{B} 2$ ). The $\alpha \mathrm{B} 2$ part extends into subdomain $\mathrm{SH} 2-\mathrm{C}$ and makes extensive hydrophobic contacts with it, thereby stabilizing the tandem structure. Superposition of the two subdomains revealed that both have a similar structure, although the $\mathrm{N}$ - and $\mathrm{C}$-terminal helices in $\mathrm{SH} 2-\mathrm{C}$ were longer and shorter, respectively, than in SH2-N (Fig. 1C). Because hydrophobic core residues are conserved from yeast to human (Fig. $1 A$, triangles), the tandem $\mathrm{SH} 2$ domain fold is conserved among eukaryotes.

Unique Structure of the Tandem SH2 Domain-An arrangement of two subsequent $\mathrm{SH} 2$ domains was previously observed in signaling proteins present in higher eukaryotes. Structures of four proteins with two adjacent SH2 domains were published from the related tyrosine kinases ZAP-70 $(25,26)$ and Syk $(27)$ and from the related tyrosine phosphatases SHP-2 $(28,29)$ and SHP-1 $(30)$. A comparison revealed that these structures are all strikingly different from the Spt6 tandem SH2 domain and showed different arrangements of the two SH2 domains (Fig. 2). In addition, 
A

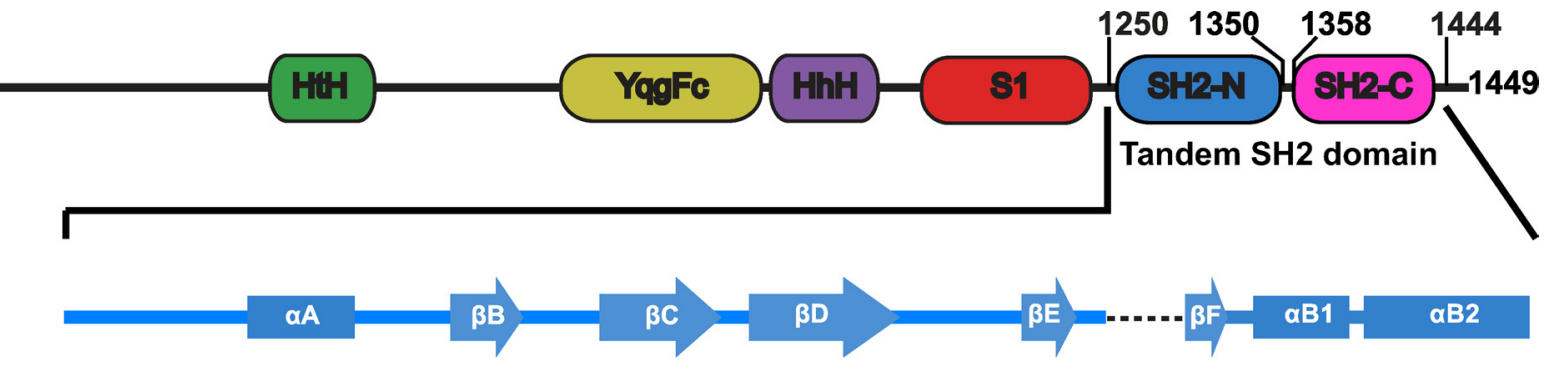

c.g. 1250 HRVINHPYYFPFNGKQAEDYLRSKERGDFVIRQSSRGDDHLAITWKLDKDLFQHVDIQELEKENPLALGKVLVVEG----1-QRYHDLDQIIVEYLQNKIRLLNELT S.c. 1251 HRVINHPYYFPFNGRQAEDYLRSKERGEFVIRQSSRGDDHLVITWKLDKDLFQHIDIQELEKFNPLALGKVLIVDN------QKYNDLDQIIVEYLQNKVRLLNEMT S.P. 1162 ARVIKHPLFKDLNASQAEAYLSKMQVGDLVIRPSSKGSDHIVVTWKVAEGSYQHIDVLELEKENEFTIGQKLLVKGRFEKMTYQYSDLDELIVLHIKAIAKKIDEMC D.m. 1332 RRVIAHPSFFNKSYAEVVAMIAEADQGEVALRPSSKSKDHLTATWKVADDIFQHIDVREEGKFNDFSLGRSLWIGT------EEEEDLDEIIARHIMPMALAARELI H.S. 1327 KRVIAHPSFHNINFKQAEKMMETMDQGDVI IRPSSKGENHLTVTWKVSDGIYQHVDVREEGKENAFSLGATLWINS------EEFEDLDEIVARYVQPMASFARDILI

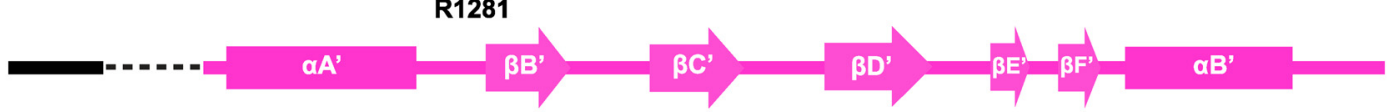

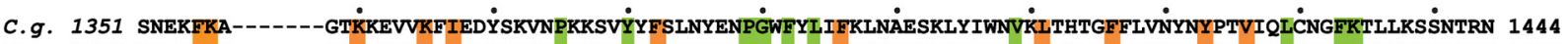
S.c. 1352 SSEKFKS------GTKKDVVKFIEDYSRVNPNKSVYYFSLNHDNPGWFYLMFKINANSKLYTWNVKLTNTGYFLVNYNYPSVIQLCNGFKTLLKSNSSKN 1445 S.p. 1269 IHDKFRK-----GTOAETEKWLESYSEANPKRSCYAFCFDHQHPGYFILCFKASVNSPVTAWPVKVIPNAFFLOGNVYGDMTALCNGFK-LLYAARTKN 1361 D.m. 1433 QYKYYKPNMVTGDENERDVMEKLLREEKANDPKKIHYFFTASRAMPGKFLLSYLP--KTKVRHEYVTVMPEGYRFRGQIEDTVNSLLRWEKEHWLDPTATP 1531 H.s. 1428 NHKYYQD--CSG--GDRKKLEELLIKTKKEKPTFIPYFICACKELPGKFILGYQP--RGKPRIEYVTVTPEGFRYRGQIFPTVNGLFRWFKDHYQDPVPGI 1522

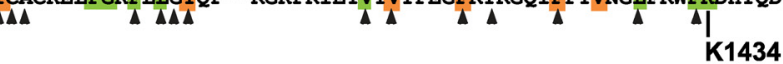

B SH2-N

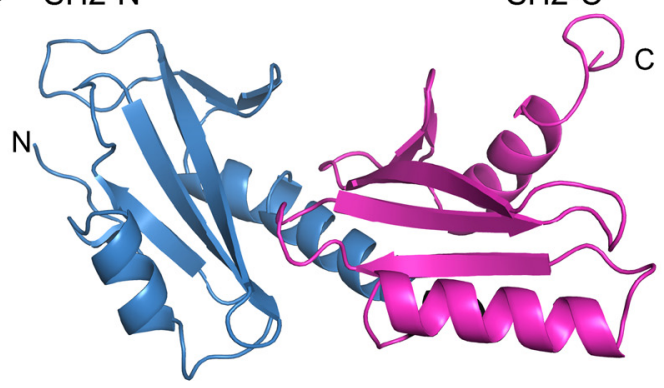

C

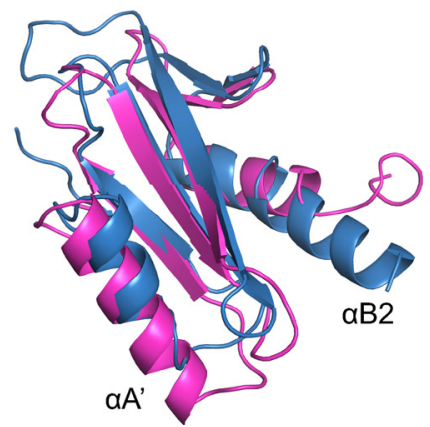

FIGURE 1. Structure of the Spt6 tandem SH2 domain. A, domain architecture of Spt6 (41) and alignment of the tandem SH2 domain amino acid sequence with corresponding regions in other eukaryotic Spt6 sequences (C. g., C. glabrata; S. C., S. cerevisiae; S. p., Schizosaccharomyces pombe; D. m., Drosophila melanogaster; H. s., Homo sapiens). Secondary structure elements are indicated above the sequences (cylinders, $\alpha$-helices; arrows, $\beta$-strands). SH2-N is colored in blue, whereas $\mathrm{SH} 2-\mathrm{C}$ is in magenta. Invariant residues are in green, whereas conserved residues are in orange. Residues in the hydrophobic core are marked with triangles. $H t H$, helix-turn-helix domain, binds to double-stranded DNA; YqgFc, predicted to be a resolvase or ribonuclease, but in Spt6, catalytic residues are exchanged, thus probably not active; $\mathrm{HhH}$, triple-helix-domain, binding to double-stranded DNA; S1, RNA-binding domain (13, 41). The sequence alignment of Spt6 tandem SH2 domains was obtained with ClustalW (42). B, ribbon representation of the Spt6 tandem SH2 domain structure. Subdomains are colored according to $A$. The left and right views are related by a $180^{\circ}$ rotation around the vertical axis. C, superposition of SH2-N and $\mathrm{SH} 2-\mathrm{C} ;$ structures were aligned by DALI-Lite (43), resulting in a root mean square deviation of $2.1 \AA$ for 83 residues. The subdomains are colored according to $A$.

the two SH2 domains are always linked by an insertion of varying structure that does not exist in the Spt6 tandem SH2 domain. Although the SH2 domains pack against each other in ZAP-70 and Syk, they do not in the SHP phosphatases and are instead positioned via additional domains present in these proteins.
The Tandem SH2 Domain Binds to the Ser-2-phosphorylated CTD in Vitro-We used fluorescence anisotropy to investigate whether the tandem $\mathrm{SH} 2$ domain binds to CTD peptides with two heptapeptide repeats (see "Experimental Procedures"). We used four peptides. One peptide was phosphorylated on both Ser-2 residues (P1), whereas another 


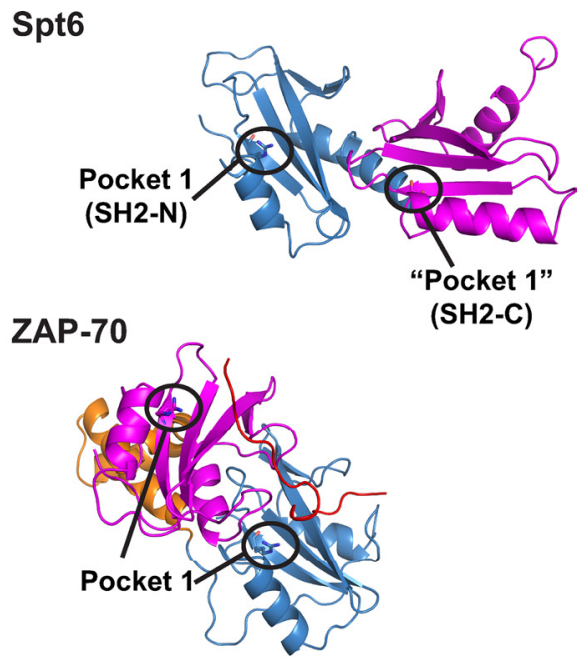

SHP-1
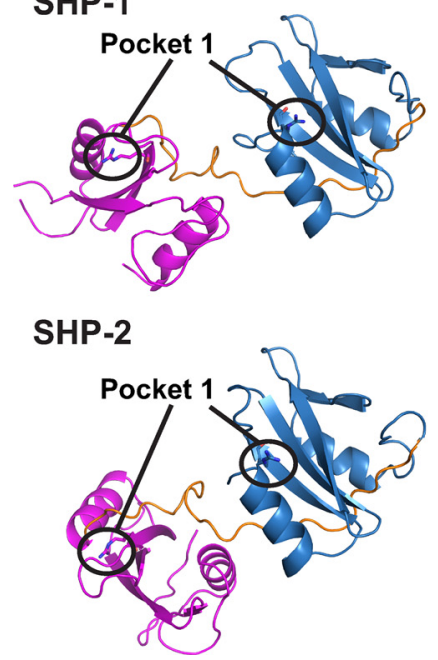

FIGURE 2. Comparison with other SH2 domain tandem arrangements. The SH2 domains of ZAP-70 (Protein Data Bank (PDB) code 2oq1), SHP-1 (PDB code 2b3o), and SHP-2 (PDB code 2shp) are shown, with their N-terminal and C-terminal SH2 domains in blue and magenta, respectively. Additional structural elements between both $\mathrm{SH} 2$ domains are represented in orange, and a bound phospho-peptide in ZAP-70 is in red. The N-terminal $\mathrm{SH} 2$ domains in these proteins were superimposed onto Spt6 subdomain $\mathrm{SH} 2-\mathrm{N}$.

one was unphosphorylated (P4). One peptide was phosphorylated only on the N-terminal Ser-2 residue (P2), and one was phosphorylated only at the C-terminal Ser-2 residue (P3). Titration equilibrium measurements revealed binding curves indicative for a specific protein-peptide interaction for the Ser-2-phosphorylated peptides P1, P2, and P3, whereas the unphosphorylated peptide P4 did not bind (Fig. $3 \mathrm{~A}$ and Table 2). Regression analysis led to a dissociation constant of $10 \mu \mathrm{M}$ for P1, and the binding affinity dropped 3-5-fold when only a single phosphorylated Ser-2 was present. Isolated $\mathrm{SH} 2-\mathrm{N}$ did not show specific binding to the same phospho-peptide (13). Thus, the tandem SH2 domain, but not SH2-N alone, binds to Ser-2-phosphorylated CTD peptides in vitro, and phosphorylation of two residues enhances binding.

CTD Binding Requires a Conserved Non-canonical Surface Crevice-The structure revealed that the canonical phosphobinding pocket in SH2-N remains accessible in the tandem domain (Fig. 3B, Pocket 1). The region in SH2-C corresponding to the canonical pocket 1 , however, lacks the conserved arginine found in all other $\mathrm{SH} 2$ domains, and instead the side chain of Tyr-1393 occupies the space of the canonical pocket. However, a conserved surface crevice was observed on the opposite side of SH2-C (Fig. 3B). This crevice is positively charged and is flanked by the invariant residue Lys-1434 that could contribute to phospho-peptide binding (Figs. $1 A$ and 3, $B$ and $C$ ).

To investigate whether $\mathrm{SH} 2-\mathrm{N}$ pocket 1 and/or the $\mathrm{SH} 2-\mathrm{C}$ crevice are required for phospho-CTD binding, we prepared three mutant variants of the tandem $\mathrm{SH} 2$ domain and tested them for binding the doubly Ser-2-phosphorylated peptide P1 (Fig. 3A). Mutation of residue Arg-1281 in pocket 1 of SH2-N to lysine led to a 1.4-fold increase of the dissociation constant. Mutation of residue Lys-1434 in the crevice of SH2-C to alanine caused a 2.5-fold increase of the dissociation constant. When both point mutations were combined into a double mutant variant of the tandem SH2 domain, binding affinity dropped strongly. Binding to the phospho-CTD peptide did not reach saturation and could not be fitted using a simple binding model. This nonsaturation behavior shows that the double mutant only interacts non-specifically with the phospho-peptide. Thus, both patches contribute to the binding affinity, and the CTD peptide apparently extends over the tandem domain surface from pocket 1 in SH2-N via a positively charged surface to the non-canonical crevice in $\mathrm{SH} 2-\mathrm{C}$ that strongly contributes to the binding affinity (Fig. 3).

We next investigated how the domain mutations influence binding of CTD peptides with single Ser-2 phosphorylations. The mutant tandem $\mathrm{SH} 2$ domain variants generally did not bind to single phosphorylated CTD peptides anymore, except for variant $\mathrm{R} 1281 \mathrm{~K}$, which bound the single phosphorylated CTD peptide P2 with almost the same affinity as the wild-type domain. This indicated that the $\mathrm{N}$-terminal phosphorylated Ser-2 residue binds to the conserved surface crevice on SH2-C. However, confirmation of this proposal and additional details of the interaction await a structure of the tandem domain in complex with a CTD peptide. Modeling indicates that a single CTD repeat cannot span the distance between the binding sites on the two $\mathrm{SH} 2$ subdomains, consistent with multiple CTD repeats contributing in the interaction.

Deletion of the Tandem SH2 Domain Causes an Elongation Defect in Vivo-Deletion of the C-terminal region in Spt6 that corresponds to the tandem $\mathrm{SH} 2$ domain in yeast causes a slow growth phenotype $(13,31)$. To investigate whether this phenotype goes along with a transcription elongation defect, we tested the sensitivity of this deletion strain to 6-AU. Sensitivity to this nucleotide-depleting drug is observed in many elongation factor mutants (32), including mutants of the Spt6 gene $(4,33)$. We observed that the deletion strain failed to grow on 6-AU-containing $(50 \mu \mathrm{g} / \mathrm{ml})$ medium (Fig. 4). This shows that the Spt6 tandem SH2 domain is essential for cell viability under conditions when transcription elongation is impaired. 
Tandem SH2 Domain in Spt6 Binds RNA Polymerase II CTD

A

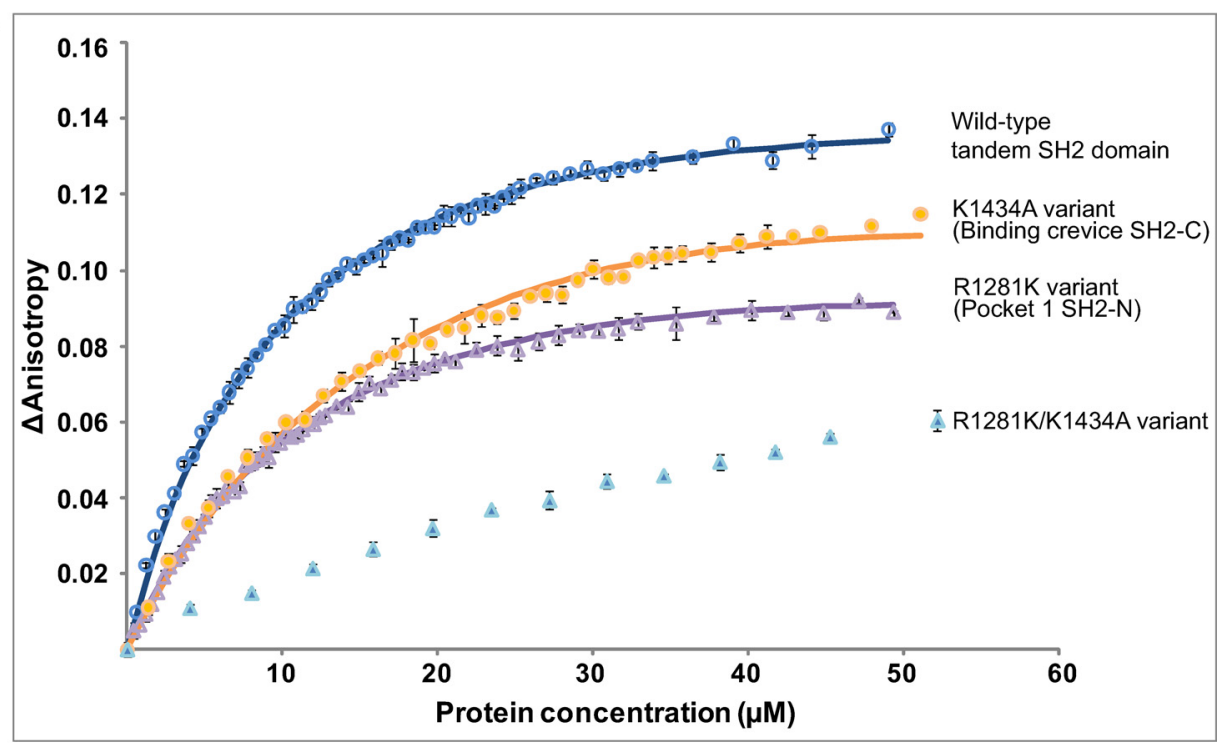

B

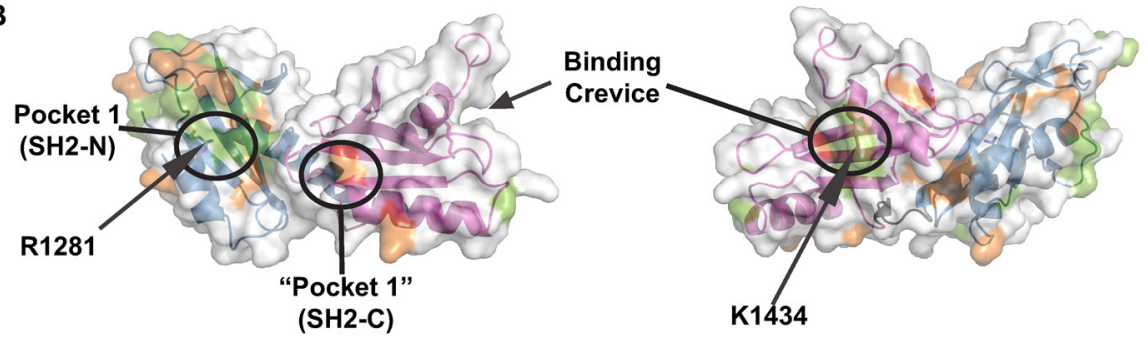

C

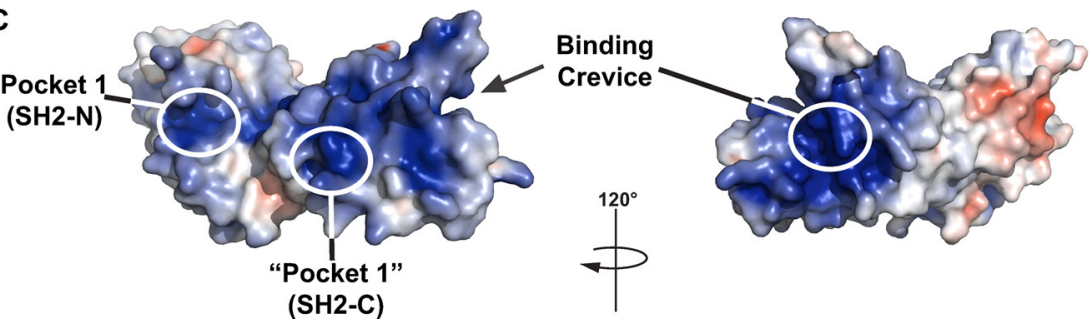

FIGURE 3. The tandem SH2 domain binds the RNA polymerase II CTD in vitro. A, fluorescence anisotropy titration of the CTD-derived phospho-peptide P1 (SYpSPTSPSYPSPTSPS; pS, phospho-serine) with different Spt6 tandem SH2 domain variants (open circles, wild-type tandem SH2 domain; open triangles, variant R1281K; filled circles, variant K1434A; filled triangles, variant R1281K/K1434A). The titration with the variant R1281K/K1434A could not be fitted with a simple binding model. The solid lines represent non-linear least square fits. B, Spt6 tandem SH2 domain surface conservation. Invariant and conserved residues are colored according to Fig. 1 . Indicated with circles are pocket 1 in $\mathrm{SH} 2-\mathrm{N}$, the region that would correspond to pocket 1 in SH2-C, and the CTDbinding crevice in SH2-C. Residues Arg-1281 and Lys-1434 are represented as sticks and are labeled. The left and right views are related by a $120^{\circ}$ rotation around the vertical axis. $C$, surface charge distribution. The domain surface is color-coded according to the electrostatic potential from negative (red) to positive (blue). Electrostatic surface potentials were calculated with APBS. The left and right views are related by a $120^{\circ}$ rotation around the vertical axis.

TABLE 2

Dissociation constants ( $\mathrm{mm}$ ) obtained by fluorescence anisotropy

$\mathrm{NF}$, not fitted, i.e. the data could not be fitted with a nonlinear least-squared regression, indicating that there is no significant binding. ND, not determined.

\begin{tabular}{lcccc}
\hline & $\begin{array}{c}\text { CTD } \\
\text { peptide } \\
\text { P1 }\end{array}$ & $\begin{array}{c}\text { CTD } \\
\text { peptide } \\
\text { P2 }\end{array}$ & $\begin{array}{c}\text { CTD } \\
\text { peptide } \\
\text { P3 }\end{array}$ & $\begin{array}{c}\text { CTD } \\
\text { peptide } \\
\text { P4 }\end{array}$ \\
\hline Tandem SH2 variant & & & & \\
WT & $10.4 \pm 0.2$ & $34.7 \pm 2.2$ & $46.0 \pm 2.3$ & NF \\
R1281K & $13.9 \pm 0.6$ & $40.7 \pm 1.1$ & NF & ND \\
K1434A & $25.7 \pm 0.7$ & NF & NF & ND \\
R1281K/K1434A & NF & NF & NF & NF \\
\hline
\end{tabular}

\section{DISCUSSION}

Here we report that the C-terminal region of the essential transcription elongation factor Spt6 contains a tandem SH2 domain that combines the previously described $\mathrm{SH} 2$ domain

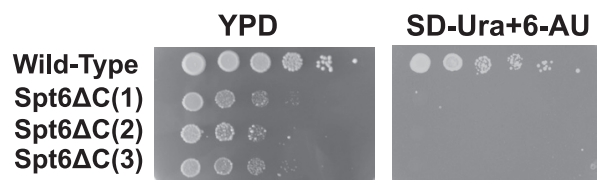

FIGURE 4. The SH2 tandem domain is apparently involved in transcription elongation in vivo. Cultures of wild-type and Spt6 tandem domain deletion yeast strains $(13,31)$ were grown in YPD medium at $30^{\circ} \mathrm{C}$ overnight and diluted to an optical density at $600 \mathrm{~nm}$ of 5 with fresh medium. The same amount of cells was spotted on plates in 10-fold serial dilutions. Plates were incubated for 5 days at $30^{\circ} \mathrm{C}$ and inspected daily. 6-AU was added to SD-Ura plates at a concentration of $50 \mu \mathrm{g} / \mathrm{ml}$.

(subdomain SH2-N) with a second, subsequent SH2 domain (subdomain SH2-C) into a single domain of novel structure. We show that the tandem $\mathrm{SH} 2$ domain, in contrast to $\mathrm{SH} 2-\mathrm{N}$ alone, binds to Ser-2-phosphorylated CTD peptides in vitro. 


\section{Tandem SH2 Domain in Spt6 Binds RNA Polymerase II CTD}

A single phosphorylated Ser-2 residue is required and sufficient for binding, but the presence of a second phosphorylated Ser-2 residue strongly enhances binding, consistent with published data (34-36). The tandem domain is also required for cell growth in the presence of the nucleotide-depleting drug 6-AU in vivo, indicating its importance for transcription elongation.

We further show that the tandem SH2 domain of Spt6 is unique in its structure and mode of CTD binding. Two adjacent $\mathrm{SH} 2$ domains are found in several proteins in higher eukaryotes but are arranged in a different way in known threedimensional structures (Fig. 2). Also, the tandem domain contains a canonical phospho-binding pocket only in its subdomain SH2-N, but not in its subdomain SH2-C, which lacks the invariant arginine residue found in all other SH2 domains.

Consistent with observed structural differences, the Spt6 tandem $\mathrm{SH} 2$ domain has a unique phospho-peptide binding mode. Two surface patches are involved in CTD binding, the canonical phospho-binding pocket in subdomain $\mathrm{SH} 2-\mathrm{N}$ and a non-canonical basic crevice in subdomain SH2-C that comprises an invariant lysine residue that contributes to binding. Mutating either one of these two conserved basic residues on the two CTD-binding surface patches resulted in a weaker interaction. It is likely that the $\mathrm{SH} 2-\mathrm{C}$ crevice interacts with a phospho-serine residue because a CTD peptide with a single phosphorylated Ser-2 residue binds equally well to wild-type tandem $\mathrm{SH} 2$ domain and a variant in which the $\mathrm{SH} 2-\mathrm{N}$ pocket is mutated. These results are consistent with an extended peptide-binding surface that reaches from the $\mathrm{SH} 2-\mathrm{N}$ pocket to the SH2-C crevice. Structural features that may provide specificity for CTD binding are, however, difficult to identify in the absence of a complex crystal structure.

Canonical SH2 domains bind phosphorylated target peptides via two pockets, one that recognizes the phosphorylated tyrosine side chain (pocket 1 ) and one that contacts residues C-terminal of the phospho-tyrosine (pocket 2), generally at register +3 . Pocket 2 generally contributes to the specificity of the interaction. In Spt6 SH2-N, pocket 1 is present and used for peptide binding, but the previously described pocket 2 , which was proposed to play a similar role as in other $\mathrm{SH} 2$ domains (13), is occluded in the tandem SH2 domain structure (Fig. 3B). In $\mathrm{SH} 2-\mathrm{C}$, neither pocket 1 nor pocket 2 is observed, but instead a conserved crevice on the opposite face contributes to peptide binding.

Despite all these differences between the Spt6 tandem SH2 domain and other arrangements of two SH2 domains, there is a functional analogy. Consistent with our observations, individual ZAP-70 SH2 domains do not bind the target phosphopeptide but do so when in a tandem arrangement (37). More generally, the phospho-peptide binding specificity of single $\mathrm{SH} 2$ domains is increased by the simultaneous binding of the peptide to an additional domain (38). Tandem SH2 domains therefore may generally increase target specificity due to cooperative peptide binding as suggested (39).

Recent data indicate that the tandem $\mathrm{SH} 2$ domain is required for retaining Spt6 at the end of transcribed genes, rather than recruiting it to genes. We have recently shown by high resolution genome-wide chromatin immunoprecipita- tion that Spt6 is recruited during an early transition in the 5 '-region of genes near the transcription start site before high levels of Ser-2 phosphorylation are reached (40). Deletion of the genomic region encoding the tandem $\mathrm{SH} 2$ domain lowered the level of recruitment, in particular at the $3^{\prime}$-region around the polyadenylation site, where levels of Ser-2 phosphorylation are high (40). These results indicated that Spt6 is recruited near the transcription start site with the use of a CTD Ser-2 phosphorylation-independent mechanism in vivo, but full recruitment and retaining of Spt6 at the polyadenylation site require the CTD-binding tandem $\mathrm{SH} 2$ domain.

Acknowledgments-We thank Dirk Kostrewa and other members of the Cramer laboratory for help. Part of this work was performed at the Swiss Light Source (SLS) at the Paul Scherrer Institute (Villigen, Switzerland). We thank the crystallization facility at the Max Planck Institute (MPI) for Biochemistry, Martinsried, Germany.

\section{REFERENCES}

1. Clark-Adams, C. D., and Winston, F. (1987) Mol. Cell. Biol. 7, 679-686

2. Swanson, M. S., Carlson, M., and Winston, F. (1990) Mol. Cell. Biol. 10, 4935-4941

3. Swanson, M. S., and Winston, F. (1992) Genetics 132, 325-336

4. Hartzog, G. A., Wada, T., Handa, H., and Winston, F. (1998) Genes Dev. 12, 357-369

5. Andrulis, E. D., Guzmán, E., Döring, P., Werner, J., and Lis, J. T. (2000) Genes Dev. 14, 2635-2649

6. Kaplan, C. D., Morris, J. R., Wu, C., and Winston, F. (2000) Genes Dev. 14, 2623-2634

7. Ardehali, M. B., Yao, J., Adelman, K., Fuda, N. J., Petesch, S. J., Webb, W. W., and Lis, J. T. (2009) EMBO J. 28, 1067-1077

8. Kaplan, C. D., Laprade, L., and Winston, F. (2003) Science 301, 1096-1099

9. Adkins, M. W., and Tyler, J. K. (2006) Mol. Cell 21, 405-416

10. Bortvin, A., and Winston, F. (1996) Science 272, 1473-1476

11. Kaplan, C. D., Holland, M. J., and Winston, F. (2005) J. Biol. Chem. 280, 913-922

12. Yoh, S. M., Cho, H., Pickle, L., Evans, R. M., and Jones, K. A. (2007) Genes Dev. 21, 160-174.

13. Dengl, S., Mayer, A., Sun, M., and Cramer, P. (2009) J. Mol. Biol. 389, 211-225

14. Maclennan, A. J., and Shaw, G. (1993) Trends Biochem. Sci. 18, 464-465

15. Koch, C. A., Anderson, D., Moran, M. F., Ellis, C., and Pawson, T. (1991) Science 252, 668-674

16. Pawson, T., Gish, G. D., and Nash, P. (2001) Trends Cell Biol. 11, 504-511

17. Weiner, M. P., and Costa, G. L. (1994) PCR Methods Appl. 4, S131-S136

18. Meinhart, A., Blobel, J., and Cramer, P. (2003) J. Biol. Chem. 278, 48267-48274

19. Budisa, N., Steipe, B., Demange, P., Eckerskorn, C., Kellermann, J., and Huber, R. (1995) Eur. J. Biochem. 230, 788-796

20. Kabsch, W. (1993) J. Appl. Crystallogr. 26, 795-800

21. Terwilliger, T. (2004) J. Synchrotron Radiat. 11, 49-52

22. Langer, G., Cohen, S. X., Lamzin, V. S., and Perrakis, A. (2008) Nat. Protoc. 3, 1171-1179

23. Emsley, P., and Cowtan, K. (2004) Acta Crystallogr. D Biol. Crystallogr. 60, 2126-2132

24. Adams, P. D., Afonine, P. V., Bunkóczi, G., Chen, V. B., Davis, I. W., Echols, N., Headd, J. J., Hung, L. W., Kapral, G. J., Grosse-Kunstleve, R. W., McCoy, A. J., Moriarty, N. W., Oeffner, R., Read, R. J., Richardson, D. C., Richardson, J. S., Terwilliger, T. C., and Zwart, P. H. (2010) Acta Crystallogr. D Biol. Crystallogr. 66, 213-221

25. Deindl, S., Kadlecek, T. A., Brdicka, T., Cao, X., Weiss, A., and Kuriyan, J. (2007) Cell 129, 735-746

26. Hatada, M. H., Lu, X., Laird, E. R., Green, J., Morgenstern, J. P., Lou, M., 
Tandem SH2 Domain in Spt6 Binds RNA Polymerase II CTD

Marr, C. S., Phillips, T. B., Ram, M. K., Theriault, K., Zoller, M. J., and Karas, J. L. (1995) Nature 377, 32-38

27. Fütterer, K., Wong, J., Grucza, R. A., Chan, A. C., and Waksman, G. (1998) J. Mol. Biol. 281, 523-537

28. Eck, M. J., Pluskey, S., Trüb, T., Harrison, S. C., and Shoelson, S. E. (1996) Nature 379, 277-280

29. Hof, P., Pluskey, S., Dhe-Paganon, S., Eck, M. J., and Shoelson, S. E. (1998) Cell 92, 441-450

30. Yang, J., Liu, L., He, D., Song, X., Liang, X., Zhao, Z. J., and Zhou, G. W. (2003) J. Biol. Chem. 278, 6516-6520

31. Youdell, M. L., Kizer, K. O., Kisseleva-Romanova, E., Fuchs, S. M., Duro, E., Strahl, B. D., and Mellor, J. (2008) Mol. Cell. Biol. 28, 4915-4926

32. Exinger, F., and Lacroute, F. (1992) Curr. Genet. 22, 9-11

33. Bucheli, M. E., and Buratowski, S. (2005) EMBO J. 24, 2150-2160

34. Liu, P., Kenney, J. M., Stiller, J. W., and Greenleaf, A. L. (2010) Mol. Biol. Evol. 27, 2628-2641
35. Stiller, J. W., and Cook, M. S. (2004) Eukaryotic Cell 3, 735-740

36. Liu, P., Greenleaf, A. L., and Stiller, J. W. (2008) Mol. Biol. Evol. 25, 719-727

37. Wange, R. L., Malek, S. N., Desiderio, S., and Samelson, L. E. (1993) J. Biol. Chem. 268, 19797-19801

38. Machida, K., and Mayer, B. J. (2005) Biochim. Biophys. Acta 1747, 1-25

39. Ottinger, E. A., Botfield, M. C., and Shoelson, S. E. (1998) J. Biol. Chem. 273, 729-735

40. Mayer, A., Lidschreiber, M., Siebert, M., Leike, K., Söding, J., and Cramer, P. (2010) Nat. Struct. Mol. Biol. 17, 1272-1278

41. Johnson, S. J., Close, D., Robinson, H., Vallet-Gely, I., Dove, S. L., and Hill, C. P. (2008) J. Mol. Biol. 377, 1460-1473

42. Thompson, J. D., Higgins, D. G., and Gibson, T. J. (1994) Nucleic Acids Res. 22, 4673-4680

43. Labarga, A., Valentin, F., Anderson, M., and Lopez, R. (2007) Nucleic Acids Res. 35, W6-W11 